\title{
State Space Based Linear Controller Design for the Inverted Pendulum
}

\author{
M. Kuczmann \\ Széchenyi István University, Department of Automation \\ Egyetem tér 1, H-9026, Gyốr, Hungary \\ E-mail: kuczmann@sze.hu
}

Abstract: In a previous survey paper the detailed PID controller design to stabilize the inclination angle as well as the horizontal movement of an inverted pendulum system has been presented. In this paper the linear controller design based on the state space representation is shown step by step. Pendulum model is based on Euler-Lagrange modeling, and the nonlinear state space model is linearized in the unstable upward position, finally pole placement by Ackermann formula and Bass-Gura equation, moreover linear quadratic optimal control are presented. The pendulum has been inserted into a virtual reality laboratory, which is suitable to use in model based control teaching.

Keywords: inverted pendulum, model based control, pole placement, optimal control

\section{Introduction}

This paper presents a comprehensive study of controller design for an inverted pendulum mounted on a cart which can only move horizontally. Design is based on the state space representation of the plant. PID controller design with some drawbacks has been shown in the latter paper [1].

The inverted pendulum is an unstable system that must be stabilized by the pushingpulling force $F=F(t)$ acting on the cart by an electric motor (Fig. 1), i.e. to reach 


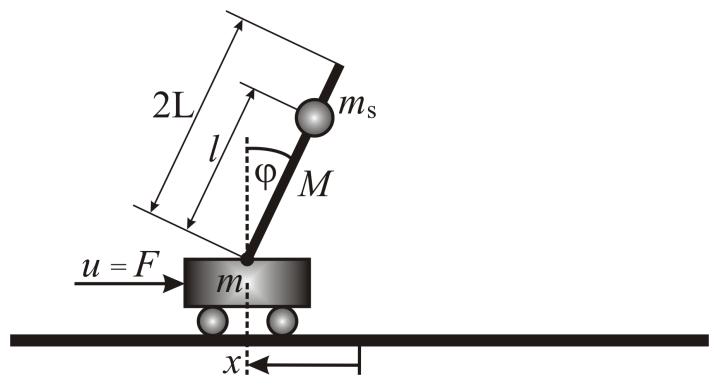

Figure 1. The inverted pendulum model.

the inclination angle $\varphi$ being zero. The pendulum simply falls over if the cart is not moved to balance it. By the presented methods, the horizontal movement can also be easily stabilized.

The studied plant is a popular example commonly found in control system textbooks and research literature [1-8]. The dynamics of the system are nonlinear as presented in the paper based on the above mentioned literature, but controller design is based on the linearized system.

The aim of this survey paper is to show the Euler-Lagrange modeling of the inverted pendulum system, then the linear state feedback controller design step by step. Pole placement technique and linear quadratic regulator are presented. The mentioned formulations are deeply studied, and the current paper can be used in teaching of model based control.

The latter paper [1] concluded that, two controllers are necessary to design for stabilizing the inverted pendulum. The design of two dependent controllers is tedious in some cases, however the state feedback controllers can solve this problem in an easy way. This is shown in this paper.

The real operating device has not built in this research, however the virtual reality based implementation has been performed which is applicable to understand the steps of control design. 


\section{Dynamic model of the pendulum}

To set up the dynamic model of pendulum, the Euler-Lagrange equation is applied,

$$
\frac{\mathrm{d}}{\mathrm{d} t} \frac{\partial K}{\partial \dot{q}_{i}}-\frac{\partial K}{\partial q_{i}}+\frac{\partial P}{\partial \dot{q}_{i}}=\tau_{i},
$$

where $K$ is the kinetic energy, $P$ is the potential energy, $q_{i}$ and $\tau_{i}$ are the generalized coordinates and the generalized torque (force), respectively. In the case of pendulum $i=1,2$, i.e. $q_{1}=x$ and $q_{2}=\varphi$, moreover $\tau_{1}=F$ and $\tau_{2}=0$.

The kinetic energy of the system is as follows:

$$
K=\frac{1}{2} m \dot{x}^{2}+\frac{1}{2} M v_{M}^{2}+\frac{1}{2} \Theta \dot{\varphi}^{2}+\frac{1}{2} m_{\mathrm{s}} v_{\mathrm{s}}^{2},
$$

with the mass of cart, $m$, the mass of rod, $M$, and the mass of the sphere $m_{\mathrm{s}}$. The inertial moment of the rod belonging to the center of mass is $\Theta=\frac{1}{3} M L^{2}$ (the length of the rod is $2 L$ ). The sphere can be moved along the rod, the distance from the shaft is $l$. The velocity of the center of mass of the rod as well as the sphere are $v_{M}$ and $v_{\mathrm{s}}$, respectively [1],

$$
v_{M}^{2}=\dot{x}^{2}+2 L C_{\varphi} \dot{x} \dot{\varphi}+L^{2} \dot{\varphi}^{2}, \quad v_{\mathrm{s}}^{2}=\dot{x}^{2}+2 l C_{\varphi} \dot{x} \dot{\varphi}+l^{2} \dot{\varphi}^{2} .
$$

The potential energy of the system is

$$
P=M g L C_{\varphi}+m_{\mathrm{s}} g l C_{\varphi},
$$

where $g$ is the gravitational acceleration. For simplicity, $S_{\varphi}=\sin \varphi$ and $C_{\varphi}=\cos \varphi$ notations are used in the paper.

After obtaining the terms in (1), and doing some manipulations, the following differential equations can be got (see [1] for a similar pendulum):

$$
\begin{aligned}
& \left(m+M+m_{\mathrm{s}}\right) \ddot{x}+\left(M L+m_{\mathrm{s}} l\right) C_{\varphi} \ddot{\varphi}-\left(M L+m_{\mathrm{s}} l\right) S_{\varphi} \dot{\varphi}^{2}=F, \\
& \left(M L+m_{\mathrm{s}} l\right) C_{\varphi} \ddot{x}+\left(M L^{2}+\Theta+m_{\mathrm{s}} l^{2}\right) \ddot{\varphi}-\left(M L+m_{\mathrm{s}} l\right) g S_{\varphi}=0 .
\end{aligned}
$$


From these equations, the following second order derivatives can be yielded:

$$
\ddot{\varphi}=\frac{-\theta_{\mathrm{R}}^{2} S_{\varphi} C_{\varphi} \dot{\varphi}^{2}+M_{\Sigma} \theta_{\mathrm{R}} g S_{\varphi}-F \theta_{\mathrm{R}} C_{\varphi}}{M_{\Sigma} \Theta_{\mathrm{R}}-\theta_{\mathrm{R}}^{2} C_{\varphi}^{2}},
$$

and

$$
\ddot{x}=\frac{\theta_{\mathrm{R}} \Theta_{\mathrm{R}} S_{\varphi} \dot{\varphi}^{2}-\theta_{\mathrm{R}}^{2} g S_{\varphi} C_{\varphi}+F \Theta_{\mathrm{R}}}{M_{\Sigma} \Theta_{\mathrm{R}}-\theta_{\mathrm{R}}^{2} C_{\varphi}^{2}} .
$$

Here the following notations have been introduced: $M_{\Sigma}=m+M+m_{\mathrm{s}}, \theta_{\mathrm{R}}=$ $M L+m_{\mathrm{s}} l, \Theta_{\mathrm{R}}=\frac{4}{3} M L^{2}+m_{\mathrm{s}} l^{2}$.

The two second order differential equations can be rewritten as four first order differential equations by introducing state variables: $x_{1}=x, x_{2}=\dot{x}, x_{3}=\varphi$, $x_{4}=\dot{\varphi}$, i.e. $x_{2}=\dot{x}_{1}$ and $x_{4}=\dot{x}_{3}$. Finally, the state space representation of the dynamic modell is the following:

$$
\begin{aligned}
& \dot{x}_{1}=x_{2}, \\
& \dot{x}_{2}=\frac{\theta_{\mathrm{R}} \Theta_{\mathrm{R}} S_{x_{3}} x_{4}^{2}-\theta_{\mathrm{R}}^{2} g S_{x_{3}} C_{x_{3}}+F \Theta_{\mathrm{R}}}{M_{\Sigma} \Theta_{\mathrm{R}}-\theta_{\mathrm{R}}^{2} C_{x_{3}}^{2}}, \\
& \dot{x}_{3}=x_{4}, \\
& \dot{x}_{4}=\frac{-\theta_{\mathrm{R}}^{2} S_{x_{3}} C_{x_{3}} x_{4}^{2}+M_{\Sigma} \theta_{\mathrm{R}} g S_{x_{3}}-F \theta_{\mathrm{R}} C_{x_{3}}}{M_{\Sigma} \Theta_{\mathrm{R}}-\theta_{\mathrm{R}}^{2} C_{x_{3}}^{2}} .
\end{aligned}
$$

This nonlinear system can be linearized in the unstable upright position, when $\varphi=0$ and $\dot{\varphi}=0$, i.e. the approximations $S_{\varphi} \cong \varphi$ and $C_{\varphi} \cong 1$ can be applied.

At the end, the linearized system can be modeled by the state space equations

$$
\dot{\mathbf{x}}=\mathbf{A x}+\mathbf{b} u,
$$

where

$$
\mathbf{A}=\left[\begin{array}{cccc}
0 & 1 & 0 & 0 \\
0 & 0 & \frac{-\theta_{\mathrm{R}}^{2} g}{M_{\Sigma} \Theta_{\mathrm{R}}-\theta_{\mathrm{R}}^{2}} & 0 \\
0 & 0 & 0 & 1 \\
0 & 0 & \frac{M_{\Sigma} \Theta_{\mathrm{R}} g}{M_{\Sigma} \Theta_{\mathrm{R}}-\theta_{\mathrm{R}}^{2}} & 0
\end{array}\right], \quad \mathbf{b}=\left[\begin{array}{c}
0 \\
\frac{\Theta_{\mathrm{R}}}{M_{\Sigma} \Theta_{\mathrm{R}}-\theta_{\mathrm{R}}^{2}} \\
0 \\
-\frac{\theta_{\mathrm{R}}}{M_{\Sigma} \Theta_{\mathrm{R}}-\theta_{\mathrm{R}}^{2}}
\end{array}\right]
$$


and $\mathbf{x}=\left[x_{1} x_{2} x_{3} x_{4}\right]^{\mathrm{T}}$ is the vector of the state variables.

For simplicity, the following notations are used:

$$
\mathbf{A}=\left[\begin{array}{cccc}
0 & 1 & 0 & 0 \\
0 & 0 & q & 0 \\
0 & 0 & 0 & 1 \\
0 & 0 & p^{2} & 0
\end{array}\right], \quad \mathbf{b}=\left[\begin{array}{c}
0 \\
\beta \\
0 \\
\alpha
\end{array}\right]
$$

The following data are used in the paper to represent the results: $m=5, M=10$, $m_{\mathrm{s}}=50,2 L=20, l=20$, in a coherent unit system, i.e. $\alpha=-0.0062, p=1.9925$, $\beta=0.1208, q=-67.1893$.

\section{Pole placement control}

The typical closed loop block diagrams of the very basic state feedback controller system is shown in Fig. 2. The block diagrams are equivalent, however both will be supplemented. Scalar-valued signals are represented by dashed line, the others are vector-valued.

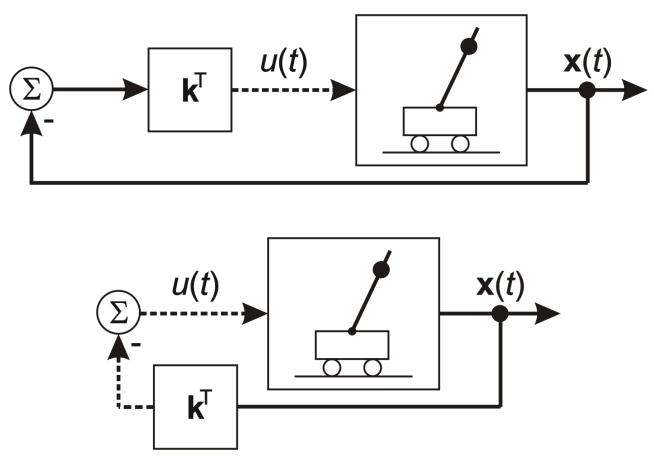

Figure 2. Block diagrams of the state feedback system.

The pendulum can be represented by the nonlinear ordinary differential equations (8), called the nonlinear model, while the linearized model is given by (9) and (10). It 
is highlighted that the controller design is based on the linearized model, however the real life pendulum is nonlinear in nature. Linear and nonlinear models are compared in the paper.

The control signal is defined as the weighted sum of the state variables, i.e.

$$
u=-\mathbf{k}^{\mathrm{T}} \mathbf{x}=-k_{1} x_{1}-k_{2} x_{2}-\cdots-k_{N} x_{N} .
$$

In this application $N=4$, and $\mathbf{k}^{\mathrm{T}}=\left[k_{1} k_{2} k_{3} k_{4}\right]$ contains the unknown parameters of the controller that must be designed.

The eigenvalues $\lambda$ of the system matrix $\mathbf{A}$ can be computed by the characteristic equation

$$
\varphi(\lambda)=|\lambda \mathbf{I}-\mathbf{A}|=\lambda^{N}+a_{1} \lambda^{N-1}+a_{2} \lambda^{N-2}+\cdots+a_{N-1} \lambda+a_{N},
$$

where $\mathbf{I}$ is the identity matrix, and the coefficients $a_{1}, a_{2}, \cdots, a_{N}$ are known building up the vector $\mathbf{a}^{\mathrm{T}}=\left[a_{1} a_{2} \cdots a_{N}\right]$. The transient behavior of the plant is depending on the eigenvalues, which can be modified by the negative feedback.

The state space representation of the closed loop system is as follows:

$$
\dot{\mathbf{x}}=\mathbf{A x}+\mathbf{b} u=\left(\mathbf{A}-\mathbf{b} \mathbf{k}^{\mathrm{T}}\right) \mathbf{x},
$$

i.e. the system matrix $\mathbf{A}$ of the open loop system is modified by the term $-\mathbf{b k}^{\mathrm{T}}$.

The eigenvalues of the closed loop system are determined by

$$
\varphi_{\mathrm{cl}}=\left|\lambda \mathbf{I}-\left(\mathbf{A}-\mathbf{b k}^{\mathrm{T}}\right)\right|=\lambda^{N}+p_{1} \lambda^{N-1}+p_{2} \lambda^{N-2}+\cdots+p_{N-1} \lambda+p_{N} .
$$

It is important to recognize, that the coefficients $p_{1}, p_{2}, \cdots, p_{N}$ are not known, because $\mathbf{k}^{\mathrm{T}}$ is unknown yet. The coefficients can be written as a vector $\mathbf{p}^{\mathrm{T}}=$ $\left[p_{1} p_{2} \cdots p_{N}\right]$.

The first step of the design is to determine the desired value of the coefficients in $\mathbf{p}^{\mathrm{T}}$ (i.e. the eigenvalues of the closed loop system), then to find the appropriate feedback gains. 
The Bass-Gura equation and the Ackermann formula are well known in the literature to design the gain vector $\mathbf{k}^{\mathrm{T}}$ [2-6].

Next, the controllability of the plant must be checked by analyzing the controllability matrix

$$
\mathbf{M}_{\mathrm{c}}=\left[\mathbf{b} \mathbf{A} \mathbf{b} \mathbf{A}^{2} \mathbf{b} \cdots \mathbf{A}^{N-1} \mathbf{b}\right] .
$$

Here $\mathbf{M}_{\mathrm{c}}$ can be computed by using the notations of (11),

$$
\mathbf{M}_{\mathrm{c}}=\left[\begin{array}{cccc}
0 & \beta & 0 & q \alpha \\
\beta & 0 & q \alpha & 0 \\
0 & \alpha & 0 & p^{2} \alpha \\
\alpha & 0 & p^{2} \alpha & 0
\end{array}\right]
$$

It is easy to see, that the matrix has four independent columns, i.e. the rank of $\mathbf{M}_{\mathrm{c}}$ is maximum. It means that, the system is controllable and state feedback methods can be applied. The inverse of $\mathbf{M}_{\mathrm{c}}$ appears in the design process.

The Bass-Gura equation looks like

$$
\mathbf{k}^{\mathrm{T}}=\left(\mathbf{p}^{\mathrm{T}}-\mathbf{a}^{\mathrm{T}}\right) \tau(\mathbf{a})^{-1} \mathbf{M}_{\mathrm{c}}^{-1},
$$

where $\tau(\mathbf{a})$ is the following Toeplitz matrix

$$
\tau(\mathbf{a})=\left[\begin{array}{cccccc}
1 & a_{1} & a_{2} & a_{3} & \cdots & a_{N-1} \\
0 & 1 & a_{1} & a_{2} & \cdots & a_{N-2} \\
\vdots & & \ddots & \ddots & \cdots & \vdots \\
\vdots & & & \ddots & & \\
0 & 0 & 0 & 0 & \cdots & 1
\end{array}\right]
$$

The Ackermann formula has the form

$$
\mathbf{k}^{\mathrm{T}}=\left[\begin{array}{llllll}
0 & 0 & 0 & \cdots & 0 & 1
\end{array}\right] \mathbf{M}_{\mathrm{c}}^{-1} \varphi_{\mathrm{cl}}(\mathbf{A}) .
$$

Of course the row vector is [0 0001$]$ in this situation, moreover

$$
\varphi_{\mathrm{cl}}(\mathbf{A})=\mathbf{A}^{N}+p_{1} \mathbf{A}^{N-1}+p_{2} \mathbf{A}^{N-2}+\cdots+p_{N-1} \mathbf{A}+p_{N} \mathbf{I} .
$$


M. Kuczmann - Acta Technica Jaurinensis. Vol.12.. No.2.. pp. 130-147, 2019
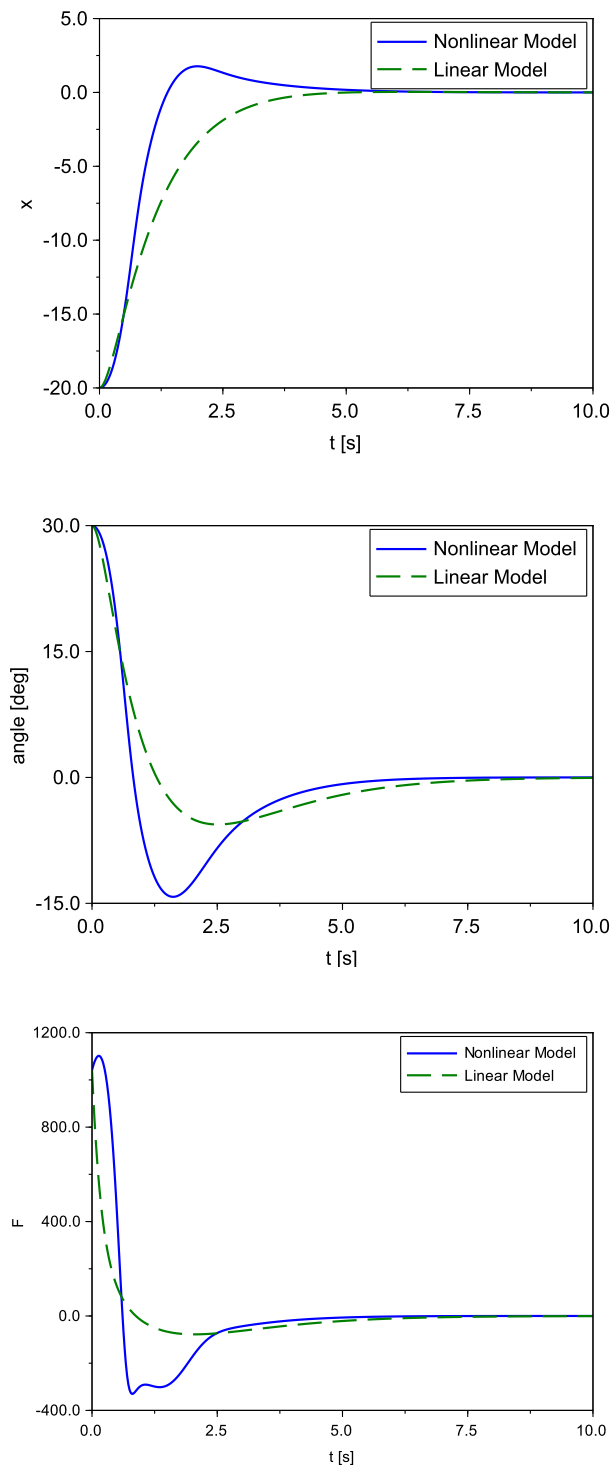

Figure 3. Stabilization of the horizontal movement (top) and the inclination angle (middle) by the control signal (bottom). 
Stabilization of the horizontal movement as well as the inclination angle can be performed easily by the pole placement methods as it is shown in Fig. 3, where the control force is also plotted. A significant difference between the linear and nonlinear model behavior can be seen, because of the large initial values. If the initial values are much smaller, then the models output are very close to each other.

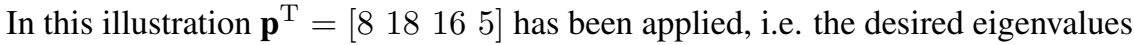
of the closed loop system are $\lambda_{1}=-5, \lambda_{2,3,4}=-1$. The plant itself is unstable with the following eigenvalues: $\lambda_{1,2}=0, \lambda_{3,4}= \pm 1.9925$, i.e. $\mathbf{a}^{\mathrm{T}}=\left[\begin{array}{lll}0 & -3.97 & 0\end{array}\right]$. The resulting feedback vector is $\mathbf{k}^{\mathrm{T}}=\left[\begin{array}{l}-81.86-261.95-5116.12-6365.03\end{array}\right]$.

Reference tracking can be realized by simple feedforward branches as shown in Fig. 4. It is noted that, there is no feedback from the output of the plant resulting in sensitive and not robust controller, however the pendulum is stabilized.
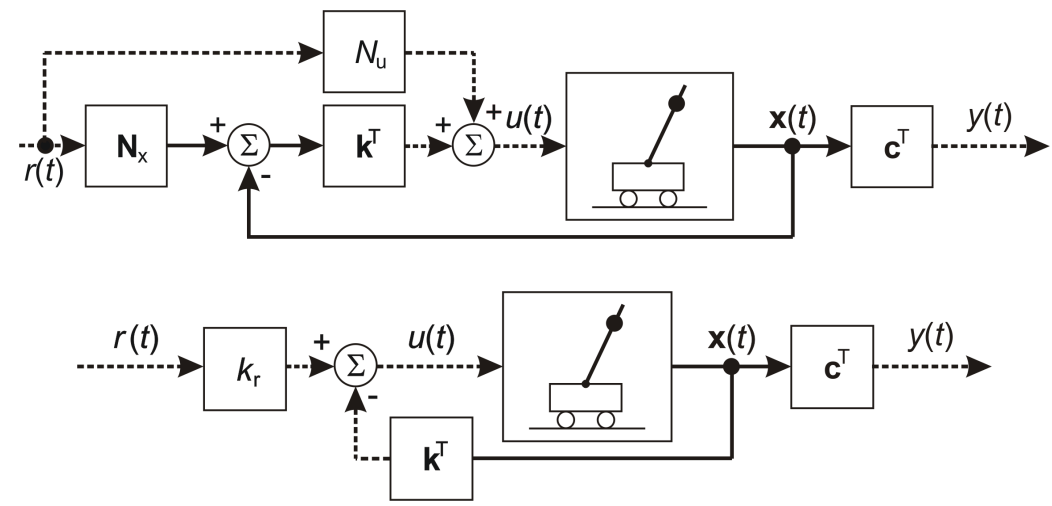

Figure 4. The state feedback system with reference trancking.

The control signal is as follows

$$
u=\mathbf{k}^{\mathrm{T}}\left(\mathbf{N}_{\mathrm{x}} r-\mathbf{x}\right)+N_{\mathrm{u}} r
$$

or

$$
u=-\mathbf{k}^{\mathrm{T}} \mathbf{x}+k_{\mathrm{r}} r
$$


in the first and second setup, respectively.

The gains in the first realization can be calculated as

$$
\left[\begin{array}{c}
\mathbf{N}_{\mathbf{x}} \\
N_{\mathrm{u}}
\end{array}\right]=\left[\begin{array}{cc}
\mathbf{A} & \mathbf{b} \\
\mathbf{c}^{\mathrm{T}} & 0
\end{array}\right]^{-1}\left[\begin{array}{l}
\mathbf{0} \\
1
\end{array}\right]
$$

and the gain

$$
k_{\mathrm{r}}=\frac{-1}{\mathbf{c}^{\mathrm{T}}\left(\mathbf{A}-\mathbf{b k}^{\mathrm{T}}\right)^{-1} \mathbf{b}}
$$

is applied in the second block diagram.

Fig. 5 shows a simple reference tracking problem by the pendulum. The cart is at $x=-10$ at the initial state, where $\varphi=30^{\circ}$. The reference signal is jumping from -10 to +10 , then, after $10 \mathrm{~s}$ it is jumping back to the original state. The cart is following the reference signal, moreover the pendulum is stabilized. Controller gains are the same as above, feedforward gains are set as mentioned in (24) and (25).
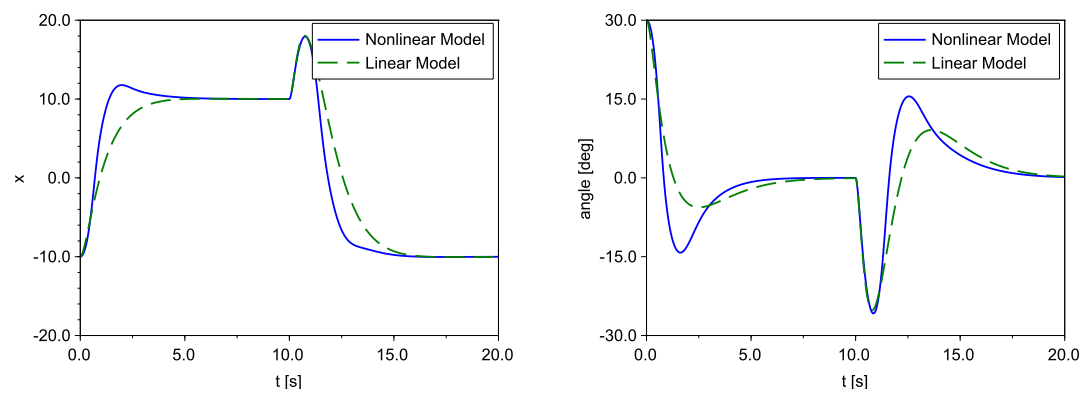

Figure 5. Reference signal tracking.

Reference tracking properties can be improved by a feedback including an integrator as shown in Fig. 6. The variable $z=z(t)$ is a new state variable, and the augmented 
system representation is as follows:

$$
\left[\begin{array}{c}
\dot{\mathbf{x}} \\
\dot{z}
\end{array}\right]=\left[\begin{array}{cc}
\mathbf{A} & \mathbf{0} \\
\mathbf{c}^{\mathrm{T}} & 0
\end{array}\right]\left[\begin{array}{l}
\mathbf{x} \\
z
\end{array}\right]+\left[\begin{array}{l}
\mathbf{b} \\
0
\end{array}\right] u,
$$

where the control signal is

$$
u=-\left[\begin{array}{ll}
\mathbf{k}^{\mathrm{T}} & k_{\mathrm{I}}
\end{array}\right]\left[\begin{array}{l}
\mathbf{x} \\
z
\end{array}\right] .
$$

Here $k_{\mathrm{I}}=N_{\mathrm{u}}$ and $k_{\mathrm{I}}=k_{\mathrm{r}}$, respectively (see notations in Fig. 6). The augmented vector $\left[\begin{array}{ll}\mathbf{k}^{\mathrm{T}} & k_{\mathrm{I}}\end{array}\right]$ can be calculated by the Bass-Gura or the Ackermann formula.
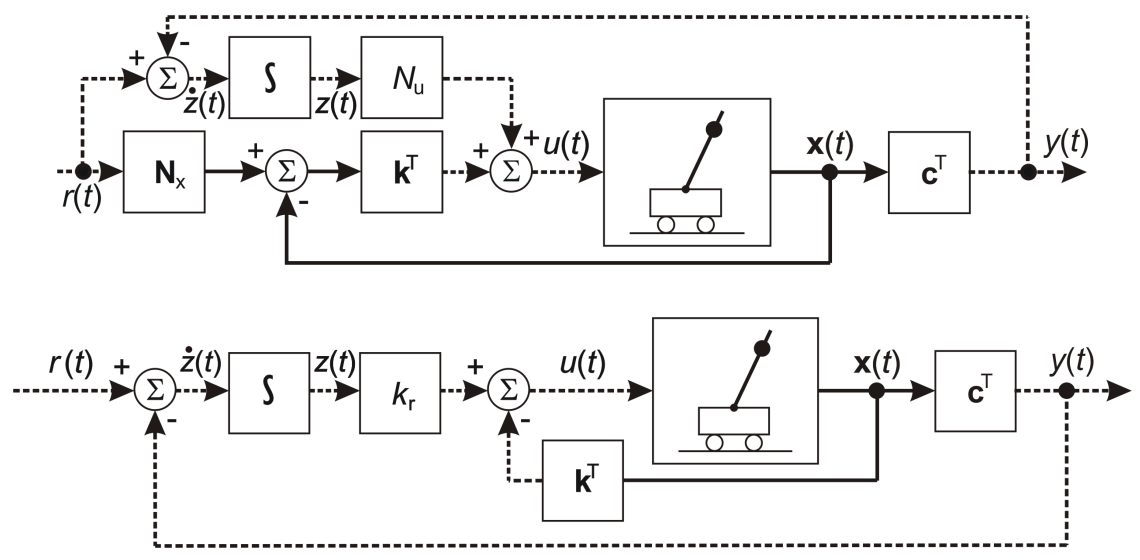

Figure 6. The state feedback system with integrator and reference trancking.

The mentioned pole placement techniques require to measure all the states. The horizontal movement and speed, moreover the inclination angle and angular speed must be measured in this case. It is easy to realize, however, in many cases it is not possible to pick up all the states, that is why state observers are designed. State observers estimate the states from measurements of the input and output of the real system as it is shown in Fig. 7. 

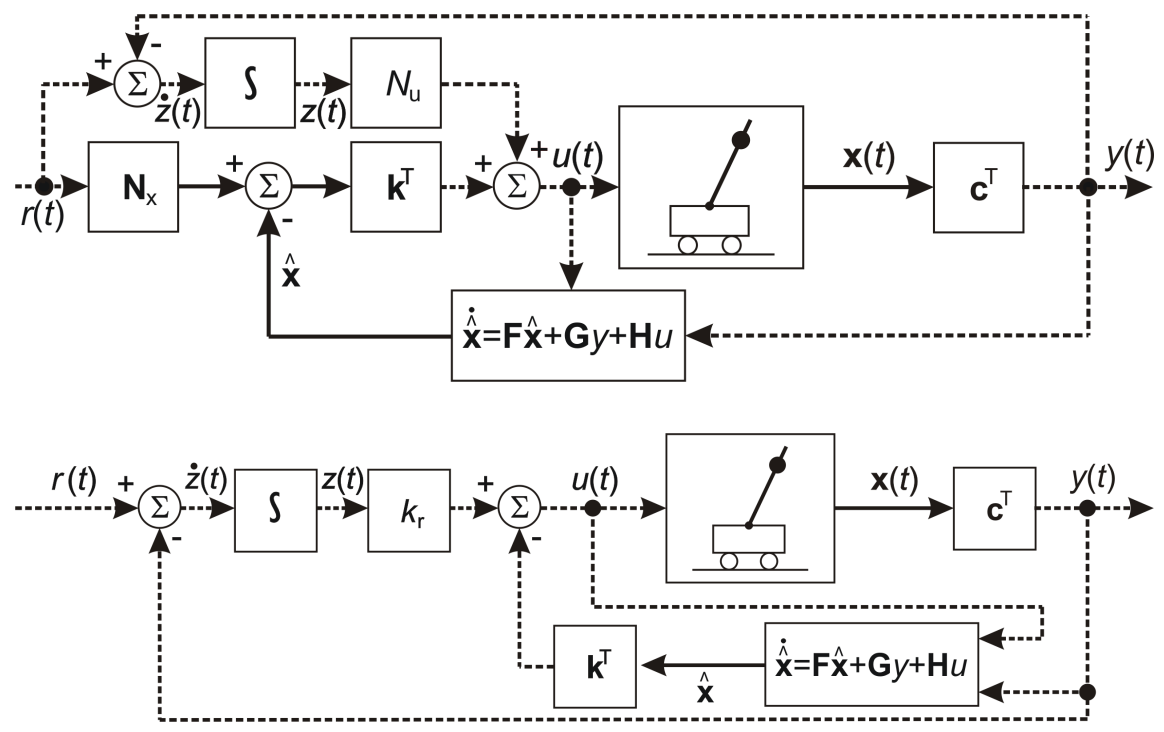

Figure 7. Block diagram of the state feedback system with observer.

The pole placement and observer design are dual to each other. The eigenvalues of the system augmented by the observer are defined by

$$
\varphi_{\mathrm{o}}=\left|\lambda \mathbf{I}-\left(\mathbf{A}-\mathbf{G c}^{\mathrm{T}}\right)\right|=\lambda^{N}+f_{1} \lambda^{N-1}+f_{2} \lambda^{N-2}+\cdots+f_{N-1} \lambda+p_{N} .
$$

The coefficients $f_{1}, f_{2}, \cdots, f_{N}$ can be written as a vector $\mathbf{f}^{\mathrm{T}}=\left[f_{1} f_{2} \cdots f_{N}\right]$.

The first step of the observer design is to determine the desired value of these coefficients (i.e. the eigenvalues), then to find the appropriate observer gain $\mathbf{G}$. The Bass-Gura equation and the Ackermann formula can also be applied. 
Next, the observability of the plant must be checked, i.e. the observability matrix

$$
\mathbf{M}_{\mathrm{o}}=\left[\begin{array}{l}
\mathbf{c}^{\mathrm{T}} \\
\mathbf{c}^{\mathrm{T}} \mathbf{A} \\
\mathbf{c}^{\mathrm{T}} \mathbf{A}^{2} \\
\vdots \\
\mathbf{c}^{\mathrm{T}} \mathbf{A}^{N-1}
\end{array}\right]
$$

must have full rank. It can only be reached if the horizontal movement of the cart is measured, i.e.

$$
\mathbf{c}^{\mathrm{T}}=\left[\begin{array}{llll}
1 & 0 & 0 & 0
\end{array}\right], \quad D=0,
$$

otherwise the rank of the observability matrix is less then four. The full rank observability matrix is according to the notations of (11) is

$$
\mathbf{M}_{\mathrm{o}}=\left[\begin{array}{llll}
1 & 0 & 0 & 0 \\
0 & 1 & 0 & 0 \\
0 & 0 & q & 0 \\
0 & 0 & 0 & q
\end{array}\right] .
$$

Duality means a simple change of the matrices: $\mathbf{A} \rightarrow \mathbf{A}^{\mathrm{T}}, \mathbf{b} \rightarrow \mathbf{b}^{\mathrm{T}}, \mathbf{M}_{\mathrm{c}} \rightarrow \mathbf{M}_{\mathrm{o}}^{\mathrm{T}}$, $\mathbf{k} \rightarrow \mathbf{G}$, with which the Bass-Gura equation has the form

$$
\mathbf{G}^{\mathrm{T}}=\left(\mathbf{f}^{\mathrm{T}}-\mathbf{a}^{\mathrm{T}}\right) \tau(\mathbf{a})^{-1} \mathbf{M}_{\mathrm{o}}^{-\mathrm{T}},
$$

while the Ackermann equation is given as

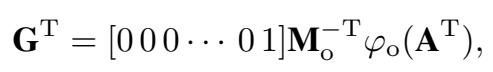

then the other terms of the observer are determined by

$$
\mathbf{F}=\mathbf{A}-\mathbf{G c}^{\mathrm{T}}, \quad \mathbf{H}=\mathbf{b} .
$$

Fig. 8 and Fig. 9 show comparisons between observer estimated and the measured signals in the case of small initial value and a larger one, respectively. The first one is very close to the linearized model, resulting very good observer performance. The linear observer is not advantageous when the real life problem is far from the stationary point. Observer eigenvalues have been set to $\lambda_{1,2,3,4}=-2$ in this example. 

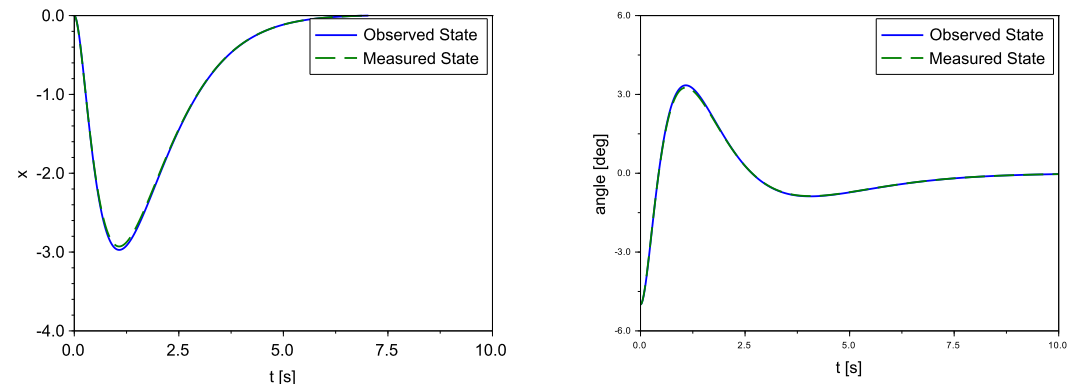

Figure 8. Comparison of observed and measured state variables $x$ and $\varphi, \varphi(0)=$ $-5^{\circ}$.
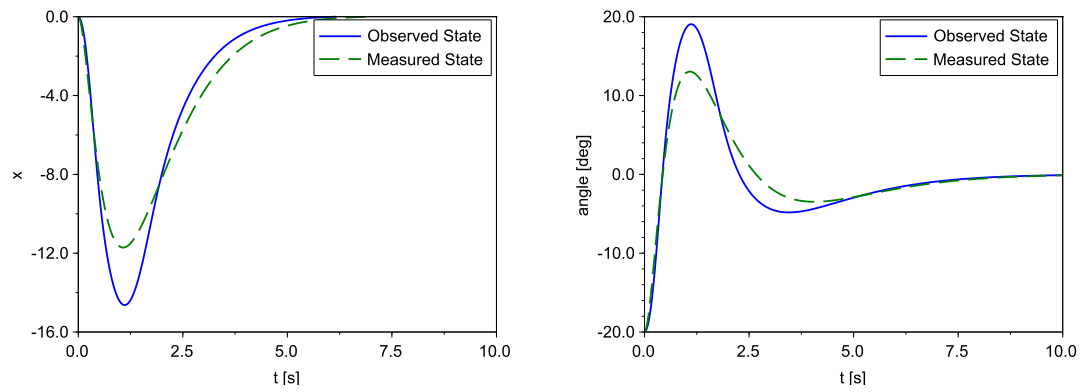

Figure 9. Comparison of observed and measured state variables $x$ and $\varphi, \varphi(0)=$ $-20^{\circ}$. 


\section{Linear quadratic optimal control}

Linear quadratic regulator (LQR) minimizes the following functional:

$$
J(\mathbf{x}, u)=\frac{1}{2} \int_{0}^{\infty}\left[\mathbf{x}^{\mathrm{T}} \mathbf{Q} \mathbf{x}+r u^{2}\right] \mathrm{d} t .
$$

The first term in the integral ensures stability $(\mathbf{x} \rightarrow \mathbf{0})$, while the second term minimizes the supply effort for the control. Matrix $\mathbf{Q}$ is symmetric and positive semidefinite, moreover $r>0$. These design parameters determine the relative importance of error in $\mathbf{x}$ and the supplied energy.

The system is defined by (9), with the initial condition $\mathbf{x}\left(t_{0}\right)$.

The optimal control law minimizing the functional (35) is a state feedback control, given by

$$
u=-\frac{1}{r} \mathbf{b}^{\mathrm{T}} \mathbf{P} \mathbf{x}=-\mathbf{k}^{\mathrm{T}} \mathbf{x}
$$

where the symmetric positive definite matrix $\mathbf{P}$ is the solution of the control algebraic Riccati equation

$$
\mathbf{P A}+\mathbf{A}^{\mathrm{T}} \mathbf{P}-\frac{1}{r} \mathbf{P b b}^{\mathrm{T}} \mathbf{P}+\mathbf{Q}=\mathbf{0} .
$$

Riccati equation can be solved by numerical techniques. The following gains have been obtained when $\mathbf{Q}=5 \mathbf{I}$ and $r=1: \mathbf{k}^{\mathrm{T}}=[-2.24-19.96-1500.66-1072.67]$. The value of $Q_{11}, Q_{22}, Q_{33}$ and $Q_{44}$ has effect on the state variable $x, \dot{x}, \varphi, \dot{\varphi}$, respectively. Control time can be decreased by increasing these values, however control signal is increased. Fig. 10 illustrates the effect of $Q_{11}$.

All the other supplementary blocks are designed in the same way presented is Section 3.

\section{Implementation}

Controller design and analysis have been realized firstly in Scilab [9]. Then the virtual reality based implementation has been performed in the frame of MaxWhere as a freely available virtual laboratory $[10,11]$. A separate future paper is planned 


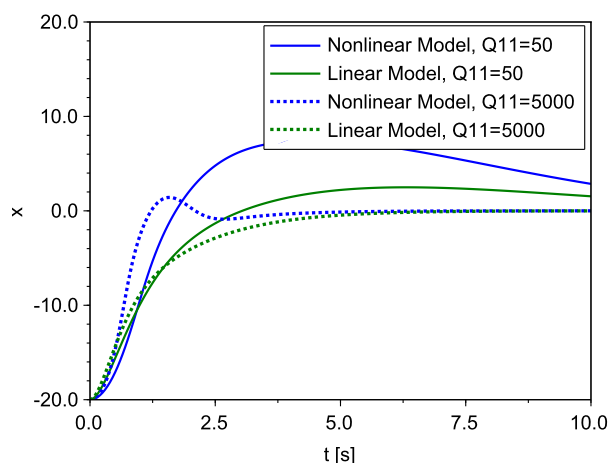

Figure 10. Effect of $Q_{11}$ on the behavior of horizontal movement.

to show the virtual lab. A snapshot about the lab can be seen in Fig. 11, where the controller settings and oscilloscopes showing some signals (e.g. the angle of pendulum, the horizontal position, the acting force versus the time) can be seen among other information like the theoretical background.

\section{Conclusion and future work}

State feedback controller design for the problem of inverted pendulum has been shown in detail in this paper. Next, nonlinear techniques [12-14] and model predictive control [15] are planned to study.

\section{Acknowledgement}

Special thank goes to Tamás Budai to include the mentioned techniques in MaxWhere. 


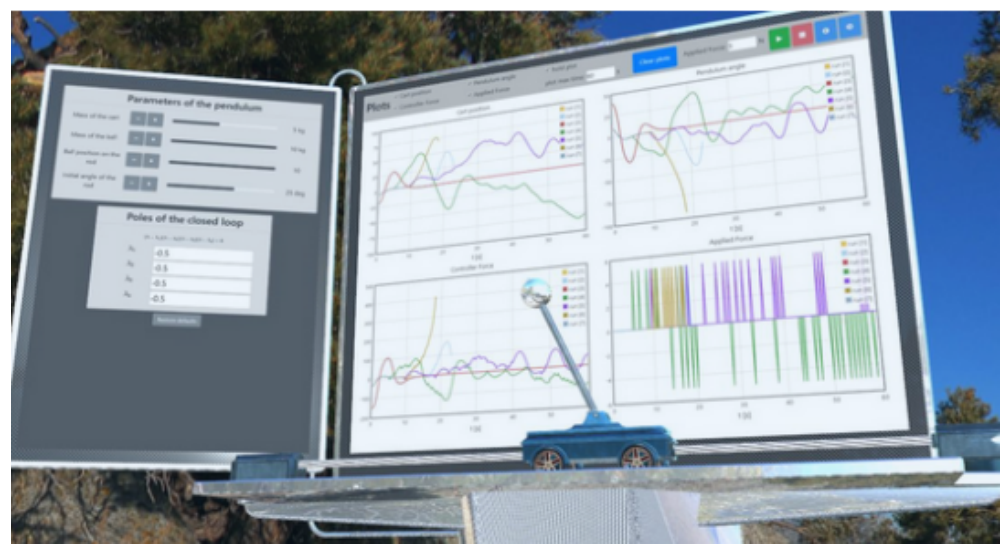

Figure 11. The inverted pendulum model in the virtual laboratory of MaxWhere.

\section{References}

[1] M. Kuczmann, Comprehensive Survey of PID Controller Design for the Inverted Pendulum, Acta Technica Jaurinensis 12 (1) (2019) pp. 55-81. doi:10.14513/actatechjaur.v12.n1.492.

[2] L. Keviczky, R. Bars, J. Hetthéssy, C. Bányász, Control Engineering, Springer, 2019.

[3] B. Lantos, Control Systems, Theory and Design I., Academic Press, Budapest, Hungary, 2009, in Hungarian.

[4] K. Ogata, Modern Control Engineering, Prentice-Hall, 1997.

[5] K. J. Aström, R. M. Murray, Feedback Systems: An introduction for scientists and engineers, Princeton University Press, 2008.

[6] P. Gáspár, J. Bokor, A. Soumelidis, An inverted pendulum tool for teaching linear optimal and model based control, Periodica Polytechnica Transportation 
Engineering 25 (1-2) (1997) pp. 9-19.

https://pp.bme.hu/tr/article/view/6592.

[7] V. A. Arya, E. G. Ashni, Stabilisation of cart inverted pendulum using the combination of PD and PID control, International Journal of Innovative Research in Science, Engineering and Technology 7 (4) (2018) pp. 3559-3565.

[8] B. Messner, D. Tilbury, Control tutorials for Matlab Simulink [cited 2019-02-23]. http://ctms.engin.umich.edu/CTMS/

[9] Scilab [cited 2019-02-23].

https://www.scilab.org/

[10] Mistems Ltd., MaxWhere [cited 2019-02-23].

https://www. maxwhere.com/

[11] T. Budai, M. Kuczmann, Development of a VR capable virtual laboratory framework, Pollack Periodica 13 (3) (2018) pp. 83-93.

doi:10.1556/606.2018.13.3.9.

[12] B. Lantos, M. Lőrinc, Nonlinear control of vehicles and robots, Springer, 2011.

[13] A. D. Drexler, Nonlinear and robust control, Budapest University of Technology and Economics, 2015, typescript in Hungarian.

[14] K. György, L. Dávid, A. Kelemen, Theoretical study of the nonlinear control algorithms with continuous and discrete-time state dependent riccati equation, Procedia Technology 22 (2016) pp. 582-591.

doi:10.1016/j.protcy.2016.01.123.

[15] W. Liuping, Model predictive control system design and implementation using Matlab, Springer, 2009. 